
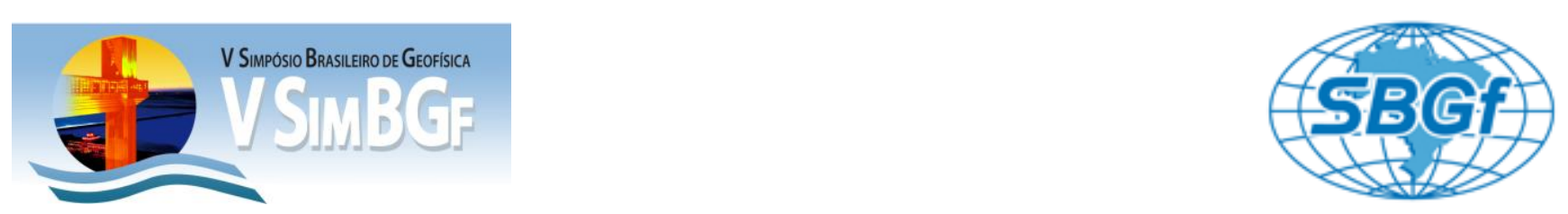

\title{
Transformações do Campo Potencial da Anomalia Magnética de Intensidade Total na região da Folha Arroio Santa Bárbara/RS: uma correlação com dados de susceptibilidade magnética
}

Cauê Vivian Hess ${ }^{1}$, Universidade Federal do Pampa; Luis Felipe de Melo Tassinari, Universidade Federal do Pampa; Guilherme Freitas Ilha, Universidade Federal do Pampa; Marcos Vinícios da S. Ferreira, Universidade Federal do Pampa; Mario J. Tomas Rosales, Universidade Federal do Pampa.

Copyright 2012, SBGf - Sociedade Brasileira de Geofísica

Este texto foi preparado para a apresentação no V Simpósio Brasileiro de Geofísica, Salvador, 27 a 29 de novembro de 2012. Seu conteúdo foi revisado pelo Comitê Técnico do V SimBGf, mas não necessariamente representa a opinião da SBGf ou de seus associados. É proibida a reprodução total ou parcial deste material para propósitos comerciais sem prévia autorização da SBGf.

\section{Abstract}

This paper deals about a methodology for integration and interpretation of aeromagnetic data, magnetic susceptibility data and topographic data (ASTER GDEM), to verify the effectiveness of the transformations of the field potential in the characterization of geological features mapped in the region of the sheet Arroyo Santa Barbara / RS . The methodology applied in this work was efficient, which makes it applicable in the verification of regional geologic mapping.

\section{Introdução}

A área de estudo situa-se a leste da cidade de Caçapava do Sul (RS) delimitada entre as Latitudes $30^{\circ} 45^{\prime} 05^{\prime \prime} \mathrm{S}$ e $30^{\circ} 57^{\prime} 33^{\prime \prime} S$ e Longitudes $53^{\circ} 56^{\prime} 11^{\prime \prime} \mathrm{W}$ e $54^{\circ} 10^{\prime} 27^{\prime \prime} \mathrm{W}$, possuindo aproximadamente $197 \mathrm{~km}^{2}$. A geologia presente é predominantemente composta por arcóseos e arcóseos líticos da Formação Maricá ( 600Ma), pelitos e arenitos muito finos a médio da Formação Santa Bárbara ( $520 \mathrm{Ma})$ e riolitos, traquitos e dacitos de coloração avermelhada da Formação Acampamento Velho, do Cambriano ( 540Ma) (Figura 1).

Afloram a noroeste e nordeste da área de estudo, respectivamente, metavulcânicas de composição andesítica associadas à metatufos e metaepiclásticas da Sequência Metamórfica Vacacaí, do Neoproterozóico ( 950 Ma) e conglomerados com clastos de vulcânicas e piroclásticas associados à Formação Hilário, do Neoproterozóico ( $580 \mathrm{Ma})$. Ao sudeste afloram basaltos de cor cinza escura da Formação Guaritas - Membro Rodeio Velho ( 480 Ma).

Afloram ainda: derrames de composição básica associados a intrusões de mesma composição, e pelitos e arenitos em camadas tabulares associados, respectivamente, às Fácies vulcânicas e pelíticas da Formação Hilário ( 580 Ma), conglomerados olimíticos e polimíticos da Formação Santa Fé ( 540 Ma) e depósitos aluvionares atuais, do Quaternário ( 65 Ma).

A base geológica do trabalho toma como fonte de referência o mapa geológico da Folha Cachoeira do Sul com escala 1: 250.000 (CPRM, 2000).

O trabalho utiliza de uma metodologia de processamento e integração de dados Aeromagnéticos, oriundos do levantamento Aerogeofísico (CPRM, 1974), conjuntamente com dados de altitude ortométrica ASTER GDEM (METI/NASA 2009) e dados de susceptibilidade magnética, visando verificar a eficácia das transformações do campo potencial para o mapeamento geológico, através do contraste das suas propriedades magnéticas, na região a nordeste da folha Arroio Santa Bárbara - RS.

Como produtos gráficos finais foram confeccionados um modelo digital de elevação do terreno (DEM), utilizando dados de altitude ortométrica ASTER GDEM, e mapas da Anomalia Magnética de Intensidade Total e das transformações do campo potencial: Amplitude do Sinal Analítico (ASA) e Gradiente Horizontal.

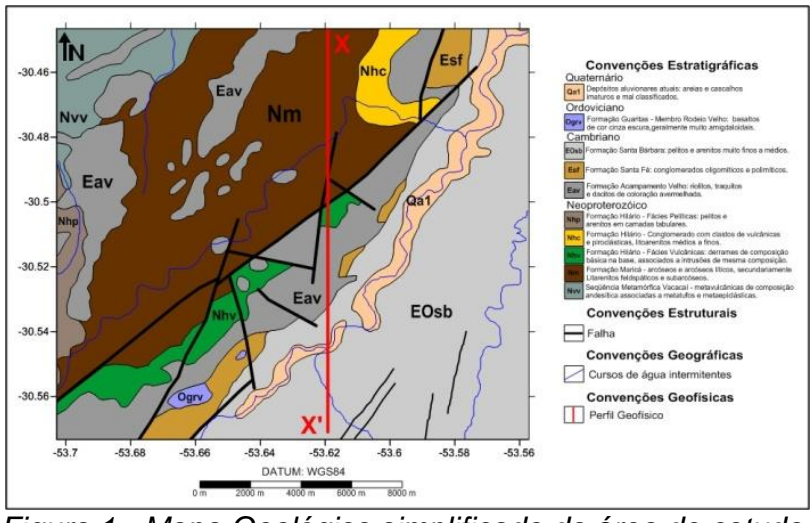

Figura 1 - Mapa Geológico simplificado da área de estudo (modificado de CPRM 2000).

\section{Modelo Digital de Elevação do Terreno}

No trabalho foram utilizados dados de altitude ortométrica da área de estudo proveniente da campanha ASTER GDEM (METI/NASA), os quais oferecem uma resolução espacial em longitude e latitude de aproximadamente 1 arc-second ( 30 metros) e uma resolução vertical de aproximadamente 7-14m (DEM accuracy - stdev).

A Figura 2 mostra o mapa topográfico da área de estudo. Posteriormente foi elaborado um Modelo Digital de Elevação do Terreno (DEM) conforme Figura 3. 


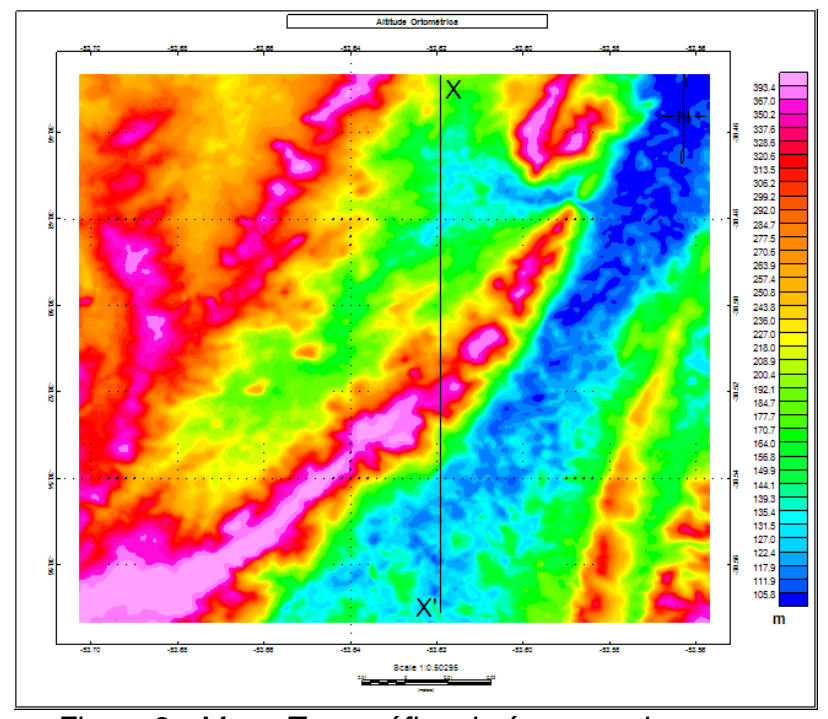

Figura 2 - Mapa Topográfico da área com base nos dados ASTER.

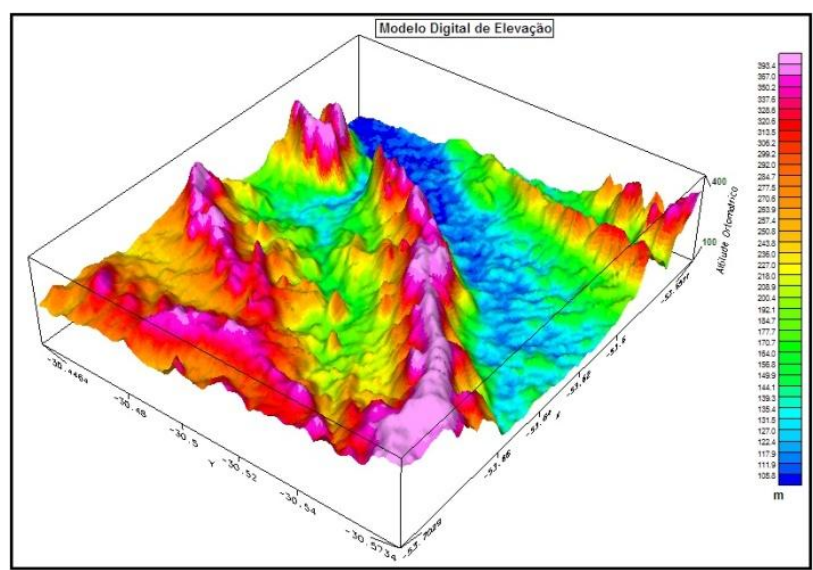

Figura 3 - Modelo Digital de Elevação do Terreno (DEM) baseados em dados ASTER.

Pode-se observar conforme a Figura 2, a presença de um vale na porção leste com valores de altitude ortométrica variando entre 100 e $140 \mathrm{~m}$ associado à Formação Santa Bárbara ( 520 Ma) e aos depósitos aluvionares atuais, do Quaternário ( $\sim 5 \mathrm{Ma})$.

$\mathrm{Na}$ porção oeste e noroeste da área os valores de altitude variam entre 200 e $400 \mathrm{~m}$ associados à Sequência Metamórfica Vacacaí ( 950 Ma), Formação Hilário ( 580 Ma) e Formação Acampamento Velho ( $540 \mathrm{Ma})$. $\mathrm{Na}$ porção central, o outro vale que observamos na Figura 2 está associado à Formação Maricá ( $600 \mathrm{Ma}) \mathrm{com}$ valores de altitude ortométrica na faixa entre 150 e $200 \mathrm{~m}$.

Vale observar que os valores mais altos de altitude ortométrica da área estão associados à Formação Acampamento Velho $(\sim 540 \mathrm{Ma})$, podendo atingir até $440 \mathrm{~m}$ de altitude em alguns pontos da área. Essa formação aflora a sudoeste da região, onde se estende na direção NE, e nas regiões oeste e sudoeste da área.
O fato dos altos topográficos da área estarem associados à Formação Acampamento Velho ( 540 Ma), formada por riolitos, traquitos e dacitos, é explicado pela erosão diferencial, já que estas rochas são mais resistatas que as outras rochas presentes na região.

\section{Anomalia Magnética de Intensidade Total}

Os dados aeromagnéticos foram compilados a partir do Levantamento Aerogeofísico (CPRM, 1974), onde foi feito um tratamento prévio dos dados com a finalidade de eliminar os ruídos ao longo das linhas de vôo, e posteriormente foi realizado um procedimento de padronização das variáveis segundo Davis (1986).

Esse tratamento consistiu na aplicação do filtro cosseno direcional nos dados já reduzidos do IGRF. Este filtro tem como função suavizar as feições em determinada direção, nesse caso, geradas pelas linhas de vôo. Além disso, aplicou-se o filtro continuação para cima de $500 \mathrm{~m}$, com o intuito de remover ruídos de alta frequência, para construção do mapa de Anomalia Magnética de Intensidade Total (Figura 4).

Analisando o mapa abaixo, pode ser observado o comportamento do campo magnético regional que varia com amplitudes aproximadas de $-55 \mathrm{nT}$ até $+40 \mathrm{nT}$, ressaltando na porção central da área um par anômalo magnético, o qual apresenta um alto ao norte e um baixo ao sul, com comprimento de onda de aproximadamente $4 \mathrm{~km}$.

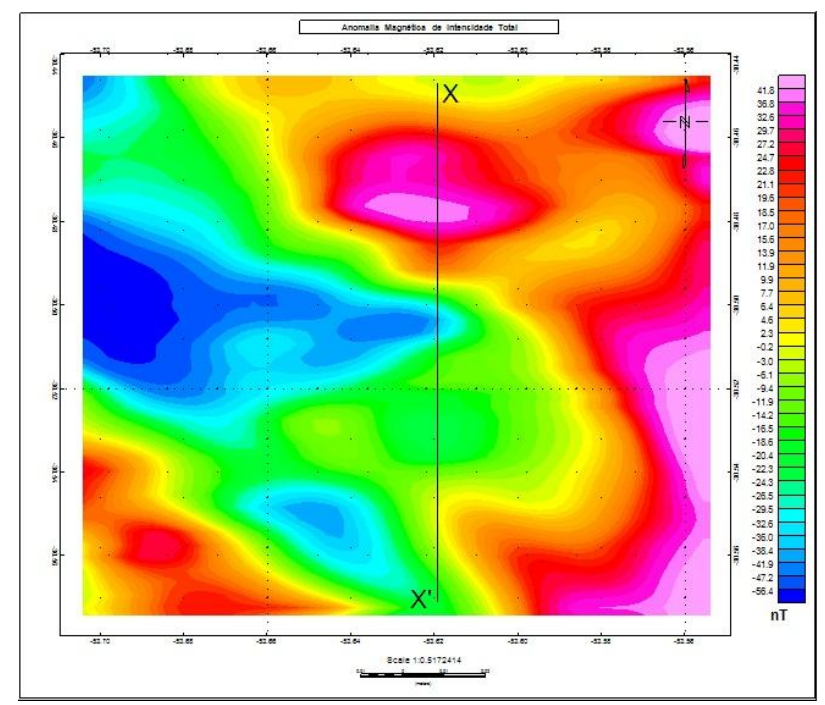

Figura 4 - Mapa da Anomalia Magnética de Intensidade Total.

\section{Transformações do Campo Potencial}

A partir do mapa residual da Anomalia Magnética de intensidade Total aplicaram-se algumas técnicas de Transformações do Campo Potencial. Tais técnicas auxiliam na análise qualitativa das características do campo magnético, permitindo assim uma melhor interpretação para a correlação litológica com estes dados. 
A Figura 5 mostra todas as transformações do campo potencial que foram realizadas, aonde se destacaram algumas na caracterização das feições geológicas que serão apresentadas a seguir.

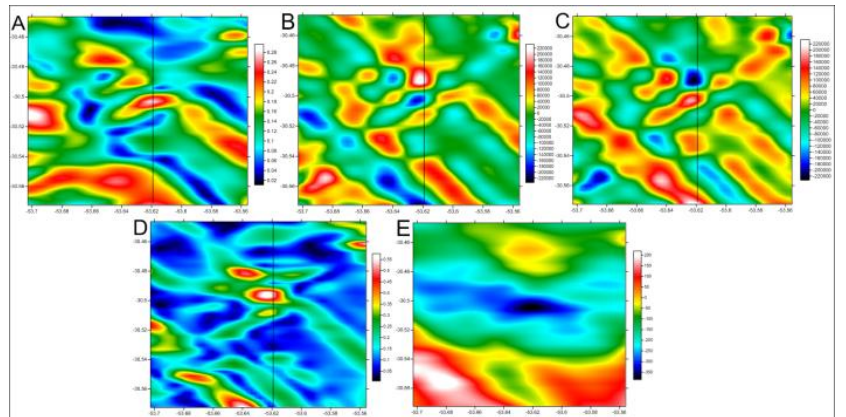

Figura 5 - Transformações do Campo Potencial da Anomalia Magnética de Intensidade Total. a) Amplitude do Sinal Analítico; b) Segunda Derivada Vertical; c) Segunda Derivada Horizontal; d) Gradiente Horizontal; e) Redução ao Pólo 90.

\section{Amplitude do Sinal Analítico}

O conceito de Sinal Analítico 2-D foi desenvolvido por Nabighian (1972, 1984). A técnica caracteriza-se por não ter dependência com relação à direção do vetor magnetização da fonte. Ela é, então, diretamente relacionada à intensidade de magnetização da fonte.

A amplitude (valor absoluto) do sinal analítico 3-D, em um ponto qualquer com coordenada $(x, y)$, pode ser obtido a partir de três gradientes ortogonais do Campo Magnético Total usando a seguinte expressão (Roest et al., 1992):

$$
|A(x, y)|=\sqrt{\left(\frac{d T}{d x}\right)^{2}+\left(\frac{d T}{d y}\right)^{2}+\left(\frac{d T}{d z}\right)^{2}}
$$

onde $|A(x, y)|$ é a Amplitude do Sinal Analítico no ponto de coordenadas $(x, y)$ e $T$ é a Anomalia Magnética de Intensidade Total no ponto de coordenadas $(x, y)$.

Segundo Roest et al., (1992), o Sinal Analítico da Anomalia Magnética de Intensidade Total reduz os dados magnéticos a anomalias cujo sinal máximo coincide com as bordas dos corpos magnetizados e cuja forma pode ser usada para determinar as profundidades destas bordas.

A Figura 6 mostra o mapa da Amplitude do Sinal Analítico da Anomalia Magnética de Intensidade Total.

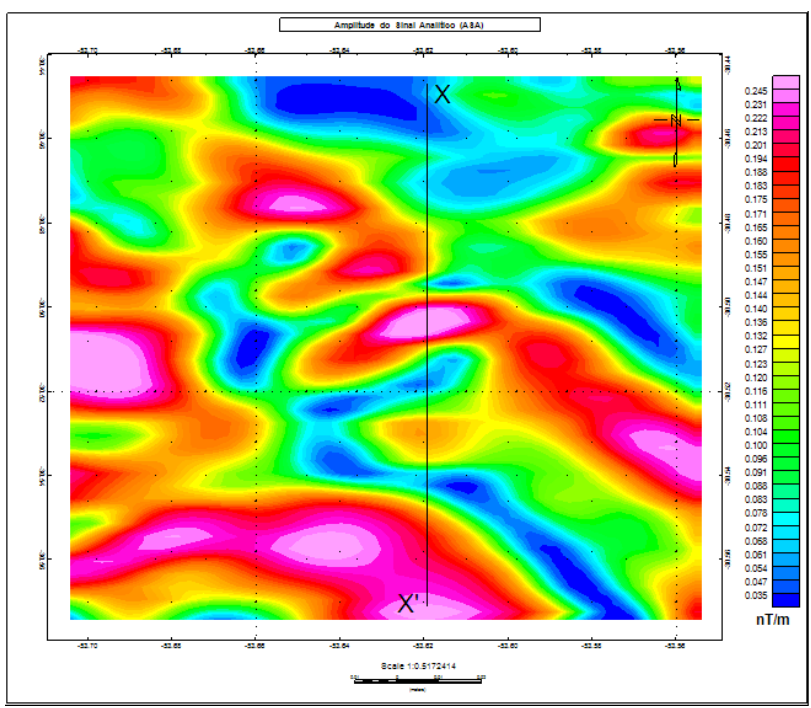

Figura 6 - Mapa da Amplitude do Sinal Analítico da Anomalia Magnética de Intensidade Total.

\section{Gradiente Horizontal}

O Gradiente horizontal do campo magnético de intensidade total foi obtido a partir de uma malha de valores de Anomalia Magnética de Intensidade Total, onde as derivadas analíticas são aproximadas por fórmulas de diferenças finitas. Essa técnica tem a característica de realçar através de máximos e mínimos, respectivamente, as bordas e os centros das fontes (Cordell \& Graunch, 1985). Ele é obtido através da seguinte equação:

$$
G(x, y)=\left[\left(\frac{d T}{d x}\right)^{2}+\left(\frac{d T}{d y}\right)^{2}\right]^{1 / 2}
$$

Onde $G(x, y)$ é o valor do Gradiente Horizontal no ponto de coordenadas $(x, y)$ e $T$ é a Anomalia Magnética de Intensidade Total nos pontos de coordenadas $(x, y)$.

A Figura 7 mostra o mapa do Gradiente Horizontal da Anomalia Magnética de Intensidade Total. 


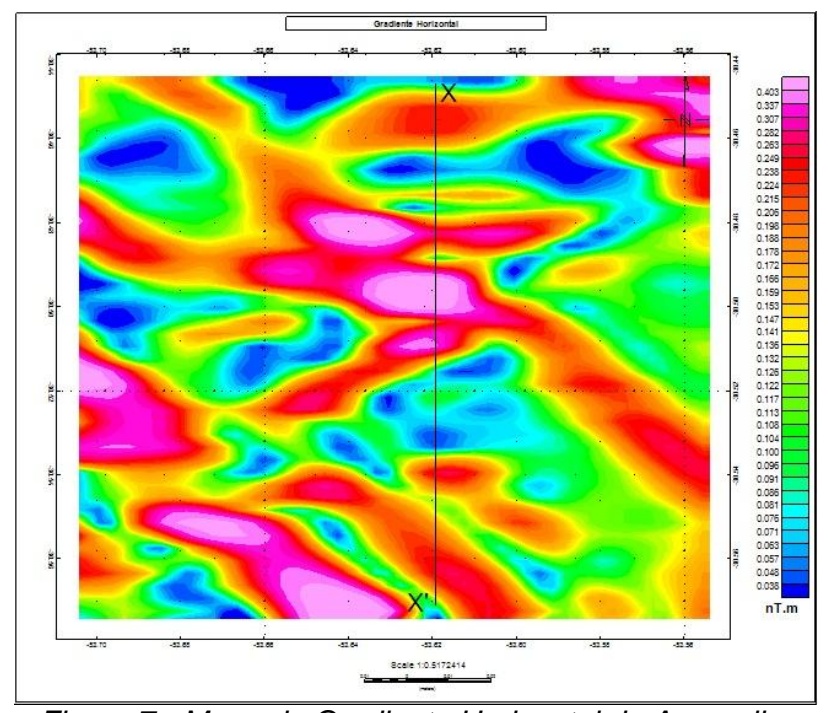

Figura 7 - Mapa do Gradiente Horizontal da Anomalia Magnética de Intensidade Total.

\section{Susceptibilidade Magnética}

Baseado no mapa da Amplitude do Sinal Analítico foi estabelecido pontos para levantamento de susceptibilidade magnética, com o intuito de verificar se o comportamento do campo magnético é condizente com a susceptibilidade magnética da área. Buscou-se realizar as medidas ao longo do perfil X-X'.

A figura 8 mostra de forma integrada o mapa da Amplitude do Sinal Analítico com a localização dos pontos amostrados.

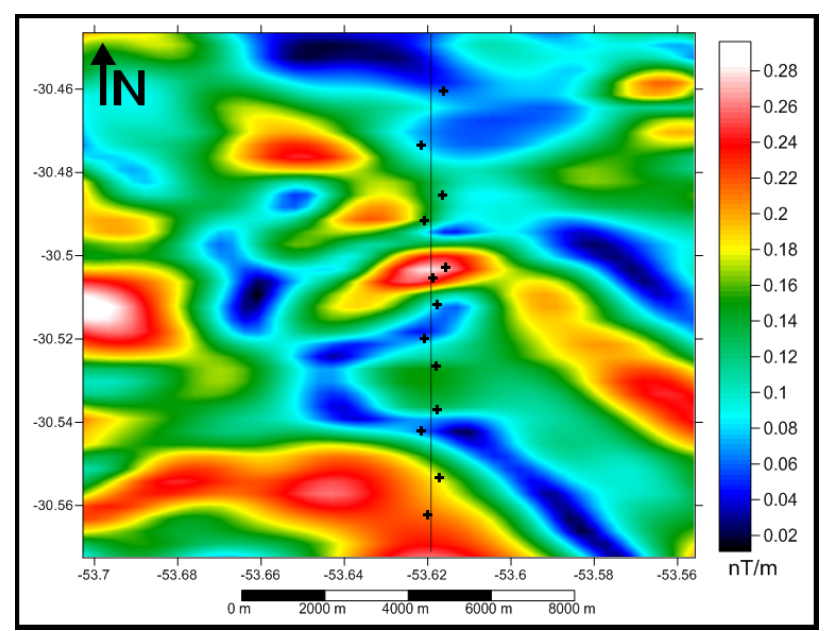

Figura 8 - Mapa da Amplitude do Sinal Analítico da Anomalia Magnética de Intensidade Total mostrando a localização das medidas de Susceptibilidade Magnética.

Para as medições de susceptibilidade magnética utilizouse 0 aparelho KT 10 Magnétic Susceptibility-Meter Terraplus cedido pela Universidade Federal do Pampa.
As medições foram feitas em diferentes amostras de quatro formações existentes ao longo do perfil X-X', onde, em cada amostra, realizou-se 08 (oito) medidas. $O$ valor final de susceptibilidade magnética de cada formação (Tabela 1) é o valor médio da média das medições de cada amostra.

Tabela 1 - Valores de Susceptibilidade Magnética

\begin{tabular}{|c|c|c|c|c|}
\hline Formação & $\begin{array}{c}\mathrm{N}^{\circ} \mathrm{de} \\
\text { Amostras } \\
\text { medidas }\end{array}$ & Latitude & Longitude & $\begin{array}{c}\mathrm{K}(10 \mathrm{E}-3) \\
\mathrm{SI}\end{array}$ \\
\hline \multirow{4}{*}{ Fm. Maricá } & \multirow{4}{*}{04} & -53.61646 & -30.46026 & \multirow{4}{*}{0,046} \\
\hline & & -53.62184 & -30.47343 & \\
\hline & & -53.61673 & -30.48526 & \\
\hline & & -53.62103 & -30.49145 & \\
\hline \multirow{2}{*}{$\begin{array}{c}\text { Fm. Hilário - } \\
\text { Fácie } \\
\text { Vulcânica }\end{array}$} & \multirow[b]{2}{*}{02} & -53.61592 & -30.50274 & \multirow{2}{*}{24,872} \\
\hline & & -53.61888 & -30.50516 & \\
\hline \multirow{3}{*}{$\begin{array}{c}\text { Fm. } \\
\text { Acampamento } \\
\text { Velho }\end{array}$} & \multirow{3}{*}{03} & -53.62184 & -30.54198 & \multirow{3}{*}{0,038} \\
\hline & & -53.61727 & -30.55301 & \\
\hline & & -53.62022 & -30.56188 & \\
\hline \multirow{4}{*}{$\begin{array}{c}\text { Fm. Santa } \\
\text { Bárbara }\end{array}$} & \multirow{4}{*}{04} & -53.61780 & -30.51161 & \multirow{4}{*}{0,093} \\
\hline & & -53.62103 & -30.51967 & \\
\hline & & -53.61807 & -30.52639 & \\
\hline & & -53.61780 & -30.53688 & \\
\hline
\end{tabular}

\section{Resultados e Discussões}

O produto final consiste em gráficos de dados integrados, onde podemos ver o comportamento da Anomalia Magnética de Intensidade Total e de suas transformações do campo ao longo do perfil X-X', conjuntamente com os dados topográficos ASTERGDEM, além da correlação com a geologia anteriormente mapeada pela folha Cachoeira do Sul (CPRM, 2000). A partir desses gráficos foi possível efetuar uma análise qualitativa dos dados, embasado pelos dados de susceptibilidade e pelos dados geológicos pré-existentes.

A Figura 9 mostra de uma maneira integrada os gráficos relacionados com o comportamento da Anomalia Magnética de Intensidade Total, da Amplitude do Sinal Analítico e do Gradiente Horizontal, conjuntamente com o relevo topográfico e a geologia ao longo do Perfil $\mathrm{X}-\mathrm{X}^{\prime}$, com orientação N-S.

\section{Perfil X - X'}

O Perfil X-X' com orientação N-S, apresenta um par anômalo magnético com intensidade que varia de +40 nT ao norte até $-40 \mathrm{nT}$ mais ao sul. Isso pode ser explicado pelo contato entre a Formação Maricá ( 600 Ma) composta por arcóseos e arcóseos líticos - e a Formação Hilário - Fácies Vulcânicas ( 580 Ma), composta por derrames de composição básica. A zona também é caracterizada por junção de falhas.

Observando o gráfico da Amplitude do Sinal Analítico da Anomalia Magnética de Intensidade Total nota-se a maior amplitude associada à Formação Hilário - Fácies Vulcânicas ( $580 \mathrm{Ma})$. Isso era justamente o que se esperava pelo fato da amplitude do sinal analítico ser diretamente relacionada à intensidade de magnetização da fonte que nesse caso é uma rocha vulcânica básica que apresentou os maiores valores de susceptibilidade (vide Tabela 1). 
A análise atenciosa do Gradiente Horizontal permite sugerir que, apesar de não conseguir delimitar bem os corpos mapeados em toda extensão da área, ao menos ao longo do perfil o gradiente demonstrou eficácia ao ressaltar as bordas e os centros dos corpos (Cordell \& Graunch, 1985).

\section{Conclusões}

O presente trabalho demonstrou a eficácia da metodologia de integração de dados aeromagnéticos, dados de susceptibilidade magnética e dados ASTERGDEM para o mapeamento geológico regional.

Verificou-se que as transformações do campo potencial realizadas na pesquisa demonstraram eficiência na identificação das feições geológicas, principalmente a Amplitude do Sinal Analítico, que apresentou alta amplitude na Formação Hilário - Fácies Vulcânicas ( 580 $\mathrm{Ma}$ ) correspondendo ao que indicam os dados de susceptibilidade magnética. O Gradiente Horizontal conseguiu realçar bem as bordas das geologias mapeadas ao longo do perfil $X-X^{\prime}$, apesar de não ter o mesmo sucesso ao longo de toda área.

O trabalho faz parte de um projeto de pesquisa em andamento que tem como objetivo geral a integração e posterior interpretação de dados oriundos de Levantamentos Aerogeofísicos e Levantamentos geofísico terrestres com a finalidade de oferecer um subsídio para o mapeamento geológico regional e esclarecer questões relacionadas com a geometria da estruturação geológica da região da Bacia do Camaquã no estado do Rio Grande do Sul.

\section{Agradecimentos}

Nossos formais agradecimentos à Universidade Federal do Pampa, por tornar possível a elaboração desse trabalho disponibilizando os instrumentos necessários. E aos nossos colegas que gentilmente nos auxiliaram nas medições terrestres.

\section{Referências}

ASTER Global DEM Validation Summary Report from http://www.gdem.aster.ersdac.or.jp/index.jsp

Blakely, Richard J.. Potential Theory in Gravity and Magnetic Applications. Cambridge University Press, 1996

Cordell, L. \& Graunch, V.J.S, 1985. Mapping basement magnetization zones from aeromagnetic data in the San Juan Basin, New Mexico. In: Hinze WJ (ed). The Utility of Regional gravity and magnetic Anomalies Maps. Society of Exploration Geophysicists, p 181-197.

CPRM - Serviço Geológico do Brasil. Geologia e Metalogênese. FOLHA SH. 22-Y-A, Estado do Rio Grande do Sul, Escala 1:250.000. Brasília: 2000. CDROM.
Davis JC. 1986. Statistics and data analysis in geology. 2nd ed., New York, John Wiley \& Sons,

Jacson, V.N.; Ramos, V.A.; Terry, S.A. \& Zuzek, A.B. Projeto Aerogeofísico Camaquã, Estado do Rio Grande do Sul Porto Alegre, Texas Instruments: Convênio Departamento Nacional da Produção Mineral/Companhia de Pesquisa de Recursos Minerais/CENEN, 208 p., 1974.

Grapher - Version 8.1.388. Graphing System. Copyright 1992 - 2009, Golden Software Inc.

Nabighian, M.N. 1972. The analytical signal of twodimensional magnetic bodies with poligonal cross-section :its properties and use for automated anomaly interpretation. Geophysics, v. 37, p. 507-517.

Nabighian, M. N., 1984. Toward a three-dimensional automatic interpretation of potential field data via generalized Hilbert transforms: Fundamental relations: Geophysics,, 49, 780-786.

Oasis Montaj, V 6.4.2 (HJ) - Standart Edition. Copyright 2007, Geosoft Inc.

Roest W.R., Verhoef J., Pilkington M. 1992. Magnetic interpretation using the 3-D analytic signal. Geophysics 57, 116125.

Surfer, Version 9.11.947 - Surface Mapping System, Copyright 1993 - 2010Golden Software, Inc. 


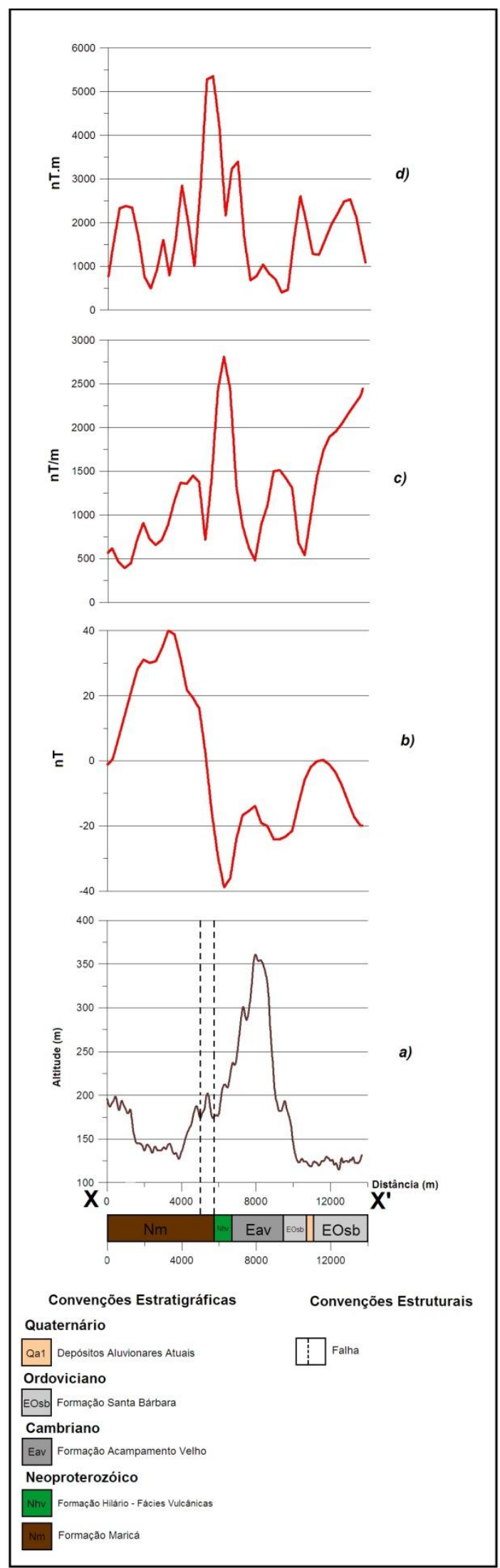

Figura 9 - Gráficos do perfil $X-X$ :

a) Relevo Topográfico baseado em dados de altitudes do modelo digital de elevação global (ASTER GDEM);

b) Anomalia Magnética de Intensidade Total;

c) Amplitude do Sinal Analítico (ASA) da Anomalia Magnética de Intensidade Total

d) Gradiente Horizontal da Anomalia Magnético de Intensidade total. 